\title{
Experimental Evaluation of Mountain Bike Suspension Systems
}

\author{
J. Titlestad, T. Fairlie-Clarke, M. Davie, A. Whittaker, S. Grant
}

\begin{abstract}
A significant distinction between competitive mountain bikes is whether they have a suspension system. Research studies indicate that a suspension system gives advantages, but it is difficult to quantify the benefits because they depend on so many variables, including the physiology and psychology of the cyclist, the roughness of the track and the design of the suspension system. A laboratory based test rig has been built that allows the number of variables in the system to be reduced and test conditions to be controlled. The test rig simulates regular impacts of the rear wheel with bumps in a rolling road. The physiological variables of oxygen consumption and heart rate were measured, together with speeds and forces at various points in the system. Physiological and mechanical test results both confirm a significant benefit in using a suspension system on the simulated rough track, with oxygen consumption reduced by around $30 \%$ and power transmitted through the pedals reduced by $30 \%$ to $60 \%$.
\end{abstract}

Keywords: mountain bike, suspension, dynamics.

\section{Introduction}

Off-road cycling, or mountain biking, has developed as an important element of the sport of cycling in the last 20 years. A significant distinction between competition bicycles is whether or nor they have a suspension system. There are three categories. A rigid frame (RF) mountain bike has no suspension. A hard tail (HT) mountain bike has a front wheel suspension only and a full suspension (SU) mountain bike has front and rear wheel suspensions. At the present time, there is a lack of information about the conditions under which a suspension system offers an advantage, the extent of the advantage and possible detrimental effects. The great majority of competition mountain bikes include a front suspension, but most professional cross-country cyclists do not ride full suspension bicycles [1]. However, research studies indicate that there can be advantages with a full suspension on rough terrain and it is of interest to explore how these can be realised under race conditions and to provide data that can be used for future design developments.

A full suspension system provides potential benefits of reduced fatigue, better traction for both up and down hill cycling and the ability to control the bicycle at faster down hill speeds [2]. It is difficult to quantify these benefits because they may depend on so many variables, including the physiology and psychology of the cyclist, the roughness of the track, the cyclist's riding style and the design of the bicycle and suspension system. Published results show definite physiological advantages for full suspension bicycles under laboratory conditions, and some advantages in controlled time trials on cross country trails. However, there is significant conflict between experimental evidence, time trials and race results. This paper reports on work [1] that has been done to help clarify the differences. The design of a laboratory based test rig that allows the number of variables in the system to be reduced and the test conditions to be controlled is described. A particular aim was to demonstrate a correlation between physiological results and mechanical dynamic measurements. The test rig allows both to be measured under sub-maximal exertion levels. The paper summarises results of this work and makes comparisons between physiological and engineering measurements.

\section{Test rig design}

\section{Past work}

Most experiments on the physiological effects of riding bicycles are carried out using standard cycle dynamometer training machines where the machine is static; there are no wheels and the cyclist pedals against a largely frictional loading. Clearly, this is not suitable for the investigation of the effect of suspension systems, and other methods must be used. A standard bicycle can be tested in the laboratory by using a power driven treadmill, usually with the treadmill inclined at a slope of about $4 \%$ to $6 \%$ so that the rider has to do work to generate a reaction force at the wheel equal to the component of the weight of the rider and bicycle acting parallel to the surface of the treadmill. Laboratory tests can also be conducted using a roller system such as used for training purposes. The rear wheel is supported by two closely spaced rollers while the front wheel runs on a single roller. It is important that the front roller be driven by the rotation of the rear rollers so that the rider can maintain balance. Experiments can also be conducted on outdoor tracks. These provide the greatest realism but the least control of conditions and restricted opportunities for measurements.

One area of interest has been the loss of energy arising because of the suspension movements induced by the cyclic variation of pedal force on a smooth road. This has been investigated by Wang and Hull [3] using treadmill tests, and results indicate that about $1.3 \%$ of the rider's power input is dissipated in the suspension system. Such results have provided the motivation for recent designs of suspension systems that minimise the response to rider induced loading. Experiments have also been conducted to investigate the effect of suspension systems on bicycles ridden over bumps. Berry et al [4] made measurements under laboratory conditions where they attached a bump to the belt of a power driven treadmill, tilted to give a $4 \%$ slope, and tested bicycles with no suspension system, with a front suspension system, with a rear suspension system and with a full suspension system. Bump impact frequency was $0.7 \mathrm{~Hz}$. Their results show that the oxygen consumed and heart rate are highest with the rigid frame bicycle and lowest with the rear only suspension system. 
The oxygen consumed when riding the full suspension bicycle over bumps was close to that measured for the rear only suspension bicycle, but heart rate was higher.

Experiments on outdoor tracks have been conducted by Seifert et al [2]. On a flat track at $16.1 \mathrm{~km} / \mathrm{h}$ and bump frequency of $0.5 \mathrm{~Hz}$, they measured the 24-hour change in creatine kinase, the volume of oxygen consumed and the heart rate. The results indicate that a significant advantage is gained over an RF bicycle by having a suspension system, but there was little difference between the HT and SU types of suspension. Time trials were also conducted over a cross-country trail, an uphill trail and a downhill trail. They found that the HT bicycle was significantly faster than the RF and SU bicycles on the cross-country trail, but there was no significant difference between bicycles in ascent and descent time trials.

MacRae et al [5] conducted up hill time trials on an 'on road' asphalt course and on an 'off road' course to compare performance of an HT bicycle and a SU bicycle. They found no significant difference in times or physiological measurements, but the power transmitted through the pedals on the SU bicycle was significantly higher than on the HT bicycle. Some increase in power transmitted is to be expected due to the heavier weight of the SU bicycle and the energy dissipated through the suspension damper, but the difference is much greater than can be explained by this and the cause is not clear.

\section{Design approach}

The results of these tests do not provide consistent data. SU bicycles perform well in laboratory tests, but HT bicycles seem to have the advantage in time trials and races. The plan for the work reported in this paper was to clarify the issues by establishing an ongoing program that would start from a simple but secure basis with a well-controlled experiment where the number of variables could be minimised. Such an experiment could be repeated with consistent outcomes and later on the constraints could be relaxed and the effect on performance of particular variables investigated. It is important to simulate accurately the inertia of the bicycle and rider and the overall resistance to forward movement in order to obtain meaningful physiological results. At the same time, the bicycle needs to be held stationary in a laboratory environment to allow accurate instrumentation and observation of the rider. A standard treadmill would not meet the inertia and resistance requirements and would leave too many unconstrained variables with the rider having to maintain balance and position on the treadmill. It was decided to design the test rig so that the rear wheel would run on a single large diameter roller while the front forks were held by a frame, but free to rotate about the front wheel axle. Bumps were fitted across the width of the roller. This arrangement allows the inertia and resistance to be simulated through the dynamics of the roller and limits the bump impact to the rear wheel, although a consequence is that the bump frequency is higher than would be experienced on most trails since it is dictated by the circumference of the roller. The subject cyclists were asked to remain in the seat and to minimise their body movement so that the measured differences between the HT and SU tests could be clearly correlated with the rear wheel impact with the bumps and the effectiveness of the suspension system in attenuating the effects of the impact.

The test rig is shown in Fig. 1. Its primary elements are a bracket to hold the front forks of the bicycle and a large diameter steel roller against which the rear wheel rotates. The inertia of the roller was set to simulate a cyclist of $74 \mathrm{~kg}$ (mean weight of subjects was $74 \pm 6.3 \mathrm{~kg}$ ) and a mountain bike of 12 $\mathrm{kg}$. This meant that impact with a bump would decelerate the roller by the same amount as the bicycle on a road would be decelerated and the subject cyclist would have to do the same amount of work to regain the speed of the roller as to regain the speed of the bicycle on the road. A strip of carpet was attached to the roller to simulate riding on a soft surface and two bumps were formed by evenly spaced rectangular wooden blocks ( $70 \mathrm{~mm}$ wide by $30 \mathrm{~mm}$ high) bolted across the roller. A range of bump sizes were tried in the preliminary testing and the chosen size provided the largest bump impact that could be applied while still allowing the cyclist to cycle at a sub-maximal level. The lower stanchions of the front forks of the bicycle were held vertical in the transverse plane and stationary so that the cyclists did not expend energy to balance the bicycle nor use their upper body to respond to front wheel impact. The bicycles were free to move on the front shock absorbers, which compress when a rear wheel impact occurs because the centre of gravity of the cyclist is between the two wheels and the inertial reaction force is therefore shared between the wheels. A resisting force was applied during the no bump tests by friction between the roller and a web strap passed over the roller and loaded by weights. The load on the web strap was set so that the time for the bicycle wheel and roller to come to a natural stop was an average of the times for the HT and SU bicycles to come to a natural stop with bumps fitted to the roller and no web strap.

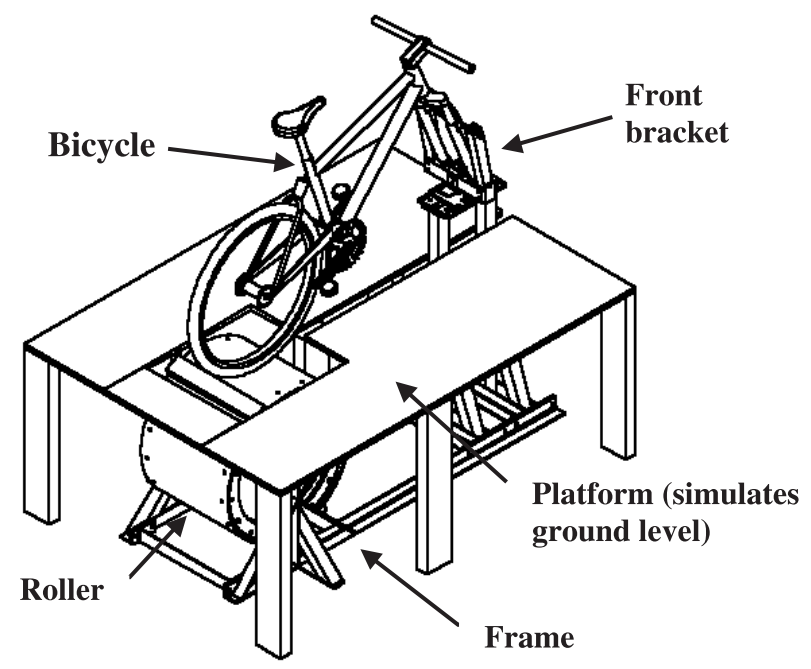

Fig. 1: The test rig arrangement

\section{The bicycles}

Two bicycles typical of those available on the market were provided on loan for the tests. The two bicycles were essentially the same apart from one having a swing arm rear suspension system (Marin Mt. Vision ${ }^{\circledR}$ ) and one having a rigid frame (Marin Rocky Ridge ${ }^{\circledR}$ ). The same front shock absorbers (Manitou Magnum ${ }^{\circledR} \mathrm{R}$ ) were attached to both bicycles with the 
setting for pre-load and damping kept constant throughout the tests. The same rear wheel was used in all the tests and the tyre pressure was kept constant at 3.4 bar.

\section{The Frame (Fig. 2)}

The frame consists of a modular welded construction to allow easy transportation, and provides a foundation to locate the roller axle and the front bracket, the position of which can be adjusted to suit bicycles of different wheelbase so that the rear axle of the bicycle remains vertically over the axle of the roller. The frame also provides some support for the platform.

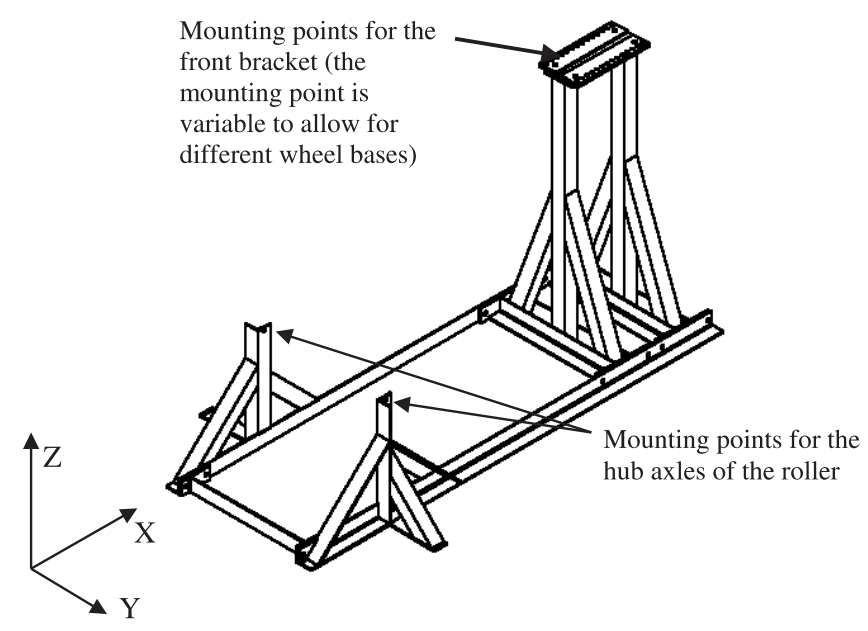

Fig. 2: The frame

\section{The Roller (Fig. 3)}

The size and mass of the roller were chosen to create an inertial effect equivalent to that of the cyclist and the bicycle. The roller is constructed out of $0.61 \mathrm{~m}$ diameter, $9 \mathrm{~mm}$ thick rolled and welded mild steel pipe. Eight tie rods are used to clamp a 24 inch bicycle wheel at each end of the roller between metal rings welded inside each end of the pipe and a second free metal ring at each end of the pipe. The two wheels thus provide the axle around which the roller rotates. The maximum run out error with this arrangement was measured at $3 \mathrm{~mm}$, giving slight undulations during the bump free tests. The total inertia of the roller assembly is $8.21 \mathrm{~kg} \cdot \mathrm{m}^{2}$.

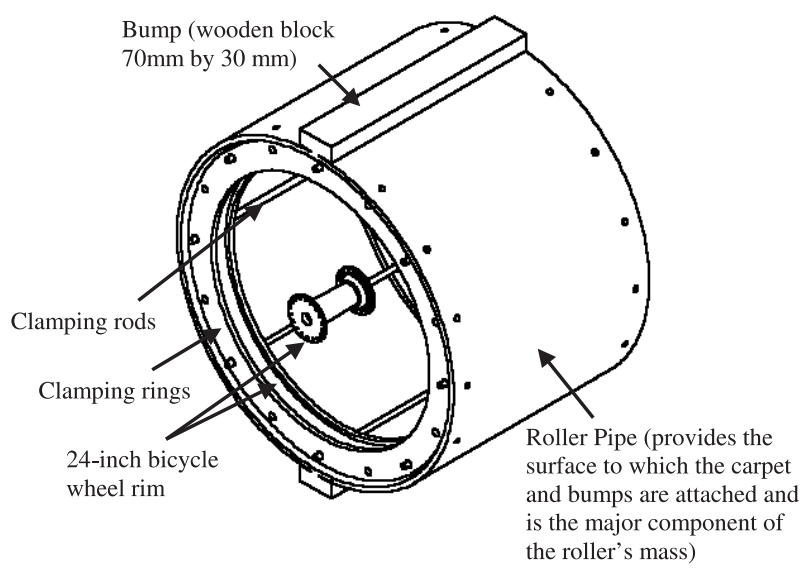

Fig. 3: The roller

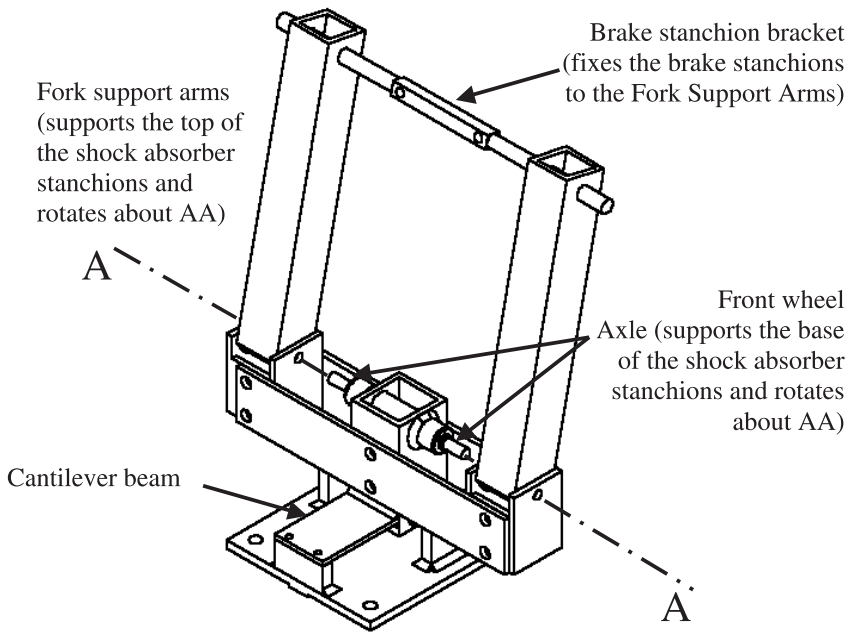

Fig. 4: The front bracket

\section{Front bracket (Fig. 4)}

The front bracket, which is fabricated from mild steel plate and square tube, holds the front wheel axle of the bicycle stationary. It also locates with the bicycle brake stanchions to provide additional transverse stiffness to ensure that the front suspension forks of the bicycle remain vertical in the transverse plane while allowing the bicycle to rotate around the front wheel axle. This arrangement allows the suspension system at the front of the bicycle to operate normally. The axle itself is mounted on a small frame that is attached to the bracket in a way that allows the horizontal reaction force at the axle to be separated from the vertical force. The frame is pivoted relative to the front bracket immediately below the front wheel axle so that vertical forces pass directly through the pivot. Horizontal forces tend to cause the frame to rotate about the pivot, but this rotation is restrained by a thin cantilever arm. A strain gauge fitted to this cantilever arm is calibrated to measure horizontal force.

\section{Instrumentation}

The saddle and handlebar accelerations were measured using linear accelerometers fitted to the seat post just under the saddle and on the top of the steerer tube of the front shock absorber. Velocity and displacements at these points were obtained by integration of the acceleration signals.

The front bracket horizontal force was measured by a strain gauge, as described above This force provides an estimate of the force between the rear tyre and the roller surface, although it is also affected by inertial loads from the movements of the cyclist.

The component of the pedal force applied perpendicular to one crank arm was calculated from the measured torque in the crank arm. The spline locking the spider and chain ring to the right crank arm and the bottom bracket axle was machined off and replaced with a bushing to allow free relative rotation. A slot was then machined into the right crank arm so that a cantilever beam could be inserted. The end of this cantilever beam was bolted to the chain rings, thus fixing the chain rings to the crank arm. All torque generated by the pedals was thus transmitted to the chain rings through the cantilever beam, which was strain gauged. 
The rotational velocity of the crank set was measured using an optical sensor with a disc attached to the chain wheel spider in place of the 22-tooth chain ring. Electronics mounted on a circuit board fitted on the base of the bottom bracket were used to convert the output frequency to a voltage proportional to the tangential velocity of the pedals. The voltage was amplified by a factor of 2000 to ensure a high signal to noise ratio when transmitted via the slip rings. The front derailleur of the bicycles was locked so that only the 32-tooth chain ring could be used. This prevented any damage to the instrumentation by accidental shifting of the gears.

A similar optical sensor and disc were used to measure the roller velocity.

A position sensor generated a signal showing when the crank passed the top position.

\section{Physiological measurements}

A Polar Favor ${ }^{\circledR}$ heart rate monitor (Polar Heart Rate Monitor, Kempele, Finland) was used to continuously monitor the heart rate of the subject, while one minute samples of expired air were collected using a two-way Hans Rudolph ${ }^{\circledR}$ 2770 mouthpiece, tubing and Douglas ${ }^{\circledR}$ bags. The expired air was analysed using a Servomex $570 \mathrm{~A}^{\circledR} \mathrm{O}_{2}$ analyser (Servomex, Crowborough, UK) and a PK Morgan ${ }^{\circledR}$ TD 801A $\mathrm{CO}_{2}$ analyser (Morgan, Rainham, UK). Both analysers were calibrated before testing with gases of known concentrations. Gas volumes were measured using a Parkinson Cowan ${ }^{\circledR}$ (Cranlea, Birmingham, UK) meter calibrated against a Tissot ${ }^{\circledR}$ spirometer (Collins, Massachusetts, USA). Standard formulae [6] were used to calculate $\mathrm{O}_{2}$ consumption and $\mathrm{CO}_{2}$ production.

\section{Experiments}

Eight male subjects participated in the tests. They were aged between 19 and 27 years and were all active in either cycling or some other physical sport. They all signed a consent form and the study was approved by the local ethics committee. Each subject was tested on both the SU and the HT bicycles with and without bumps on the roller. The first tests were conducted with bumps on the roller but without any additional braking effect. The tests were then repeated without bumps but with a brake applied to the roller and adjusted so that the time to free roll to rest was the same with and without bumps. This ensured that the workload and heart rate of the subjects during the bump tests and the no bump tests were of the same order.

The tests with each subject were conducted at the same time of day. The order in which the two bicycles were ridden was randomly assigned to the subjects. The saddle height was set so that when the pedal was at its lowest position the subject's leg was straight with the heel on the pedal. The first test included a familiarization session during which the subject was instructed to cycle at a speed between 10 and $15 \mathrm{~km} / \mathrm{h}$ that could be maintained comfortably for ten minutes. The subjects were asked to maintain this speed to within $0.5 \mathrm{~km} / \mathrm{h}$ during all their tests. To allow for different riding styles, the subjects were permitted to ride in the rear gear of their choice, but had to then use the same gear for all the tests. All the subjects wore a nose clip.

The subjects were instructed to remain motionless and not apply any load to the pedals for the first 10 seconds of the test while zero load readings were recorded. They then had the remainder of the first minute to attain their chosen test speed at the start of each test. The test proper started at the end of the first minute and continued for a further ten minutes while readings and samples were taken. The subjects were then instructed to allow the bicycle to come to a halt, to remove all load from the pedals and remain motionless for a further 10 seconds while the zero readings were checked.

Each subject was instructed to adopt a passive riding style during the tests by remaining seated on the bicycle at all times and not consciously transferring their weight. This was to ensure that the effects of the suspension were measured and not the ability of the rider to use body movements to minimise the effects of the bumps.

\section{Measurement}

Velocity, acceleration and load measurements were recorded continuously.

Heart rate was recorded 45 seconds into each minute of the test. It was found that the readings remained quite steady during the last five minutes of the test and the mean of the last two recordings was taken as an indicative value. One minute samples of expired air were taken in the ninth and tenth minutes of the test. Verbal feedback was also obtained from the subjects during the tests to indicate perceived exertion and comfort.

\section{Results}

The results of the experiments are reported in detail by Titlestad [1], and samples of these are given here in bar graph form to demonstrate the main outcomes. The measurements taken for each subject are indicated by an individual group of bars given in the order from left to right of HT and SU bicycles over bumps and then HT and SU on the smooth roller with additional resistance provided by the web brake. Figs. 5 and 6 show the physiological measurements of oxygen consumption and heart rate, while Figs. 7 and 8 show the

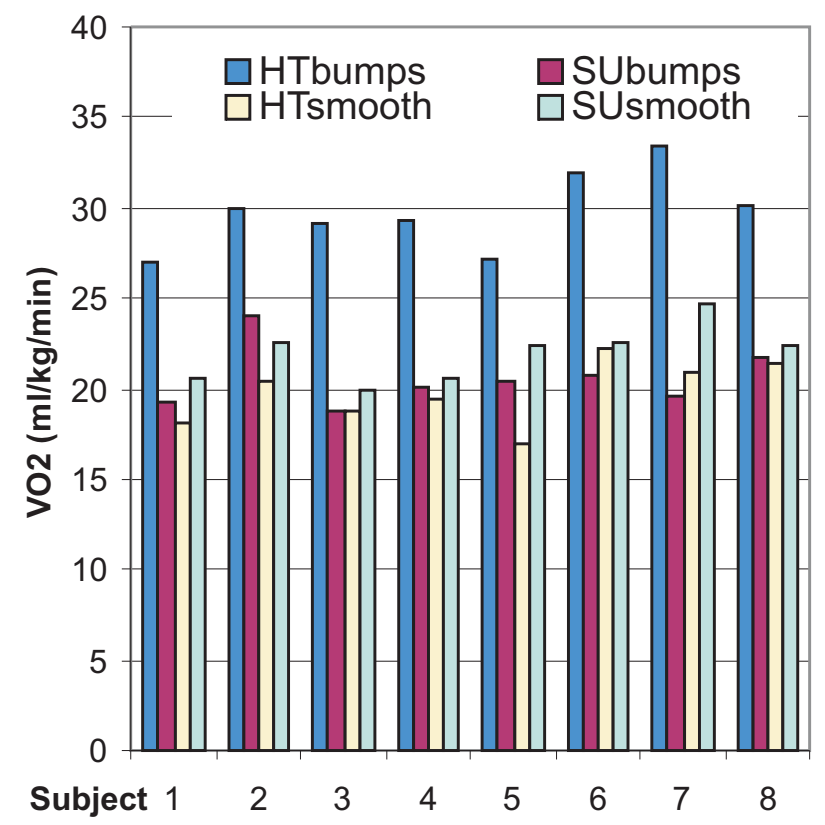

Fig. 5: Oxygen consumption 


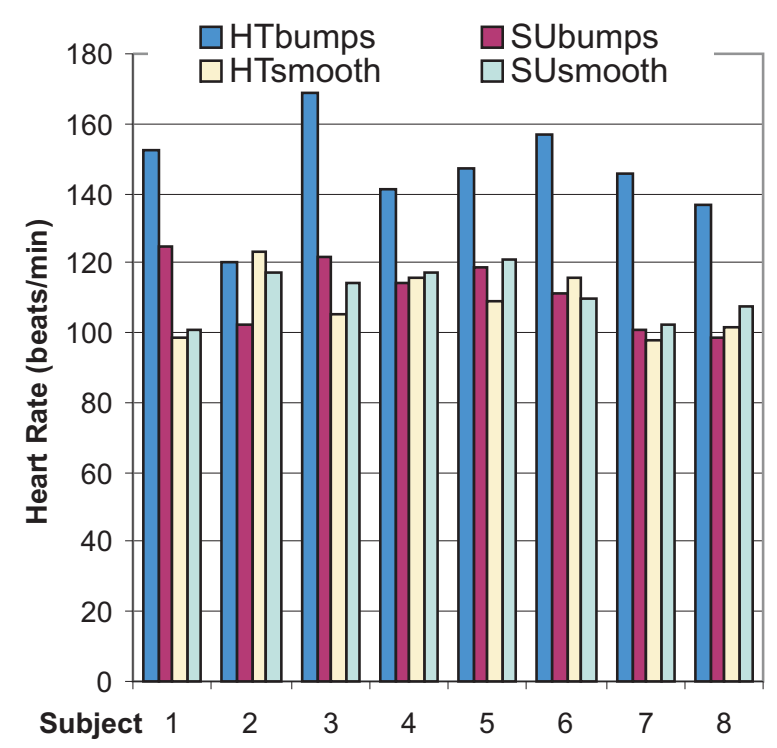

Fig. 6: Heart rate

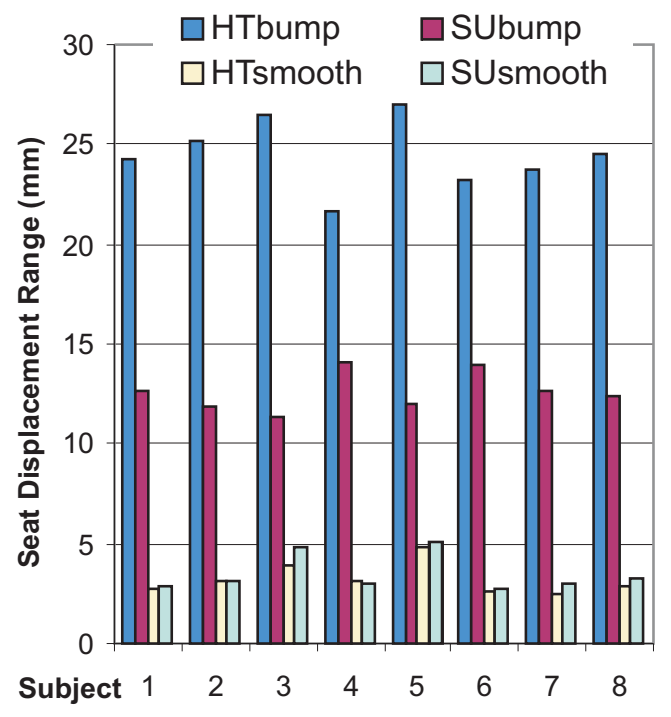

Fig. 7: Seat displacement range

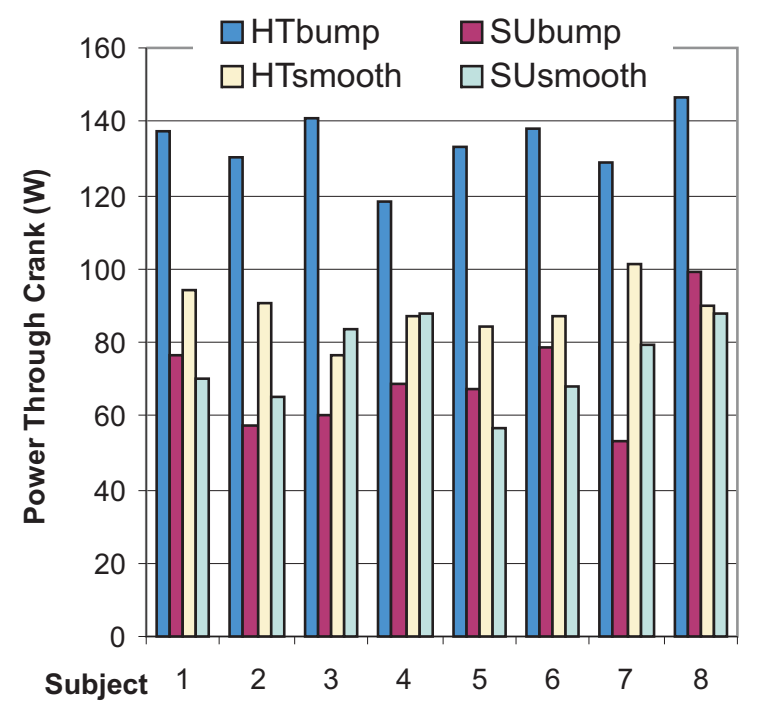

Fig. 8: Power transmitted through crank dynamic measurements. Fig. 7 shows the displacement of the seat post, derived by double integration of the acceleration data and given as the range of movement in millimetres from its highest position to its lowest position as the bicycle rides over a bump and then lands back on the roller surface. Fig. 8 shows the power being transmitted by the rider through the pedal crank arms.

For each of the variables shown in the figures, the objective is to achieve lower values that will provide an advantage for the rider. Thus, it can be seen that for the current test conditions, the SU bicycle performs better over the bumps than the HT bicycle. The physiological results show that when riding the SU bicycle the subjects consume less oxygen and their heart rates are slower. The dynamic results confirm the physiological results, with the SU bicycle rider having to transmit less power through the crank arms and thence to the rear wheel when riding over bumps. Fig. 7 also shows that there is considerable attenuation of the displacement of the seat and this will improve rider comfort.

For the tests using the smooth roller (i.e., without bumps), additional loading was applied using the web strap wrapped around the roller. This resulted in a total resistance that was higher than that experienced by the SU bicycle in the presence of bumps but lower than that experienced by the HT bicycle (note that because the HT bicycle is stiffer it experiences higher forces on impact with the bumps than the SU bicycle and therefore has a larger overall resistance). The results for the HT and SU bicycles on the smooth roller are similar to each other, with a tendency for slightly higher physiological readings from the SU bicycle. This is as expected since the suspension system reacts slightly to the varying crank arm loading due to pedalling cadence and a little energy is dissipated through the damping action of the suspension system. The seat displacement shown in Fig. 7 for the smooth roller arises primarily because of the run out error in the cylindricity of the roller, which is about $3 \mathrm{~mm}$. Marginally higher displacements occur with the SU bicycle. Power transmitted through the crank is generally, but not always, lower for the SU bicycle. It is not clear why this should be so, but it may be that some subjects rock forward and back a little so that inertia effects come into play, and there may sometimes be a back pressure applied on the second crank, which would result in an overestimate of the transmitted torque.

\section{Conclusions}

A test rig has been constructed that allows laboratory tests to investigate the effect of the impact of bicycles with bumps under controlled conditions with a minimal number of variables. A number of such tests have been completed to compare the performance of HT and SU bicycles. The test conditions represent a severely bumpy track with a high frequency of encounter with the bumps. Under these conditions, the results show a clear physiological and dynamic advantage for the SU bicycle. There is around $30 \%$ reduction in the consumption of oxygen and heart rate is reduced by between 20 to 50 beats per minute. Reduction of oxygen consumption indicates a reduced expenditure of physiological energy, and this correlates with the mechanical measurements that show a reduction of between $30 \%$ and $60 \%$ in the power transmitted through the crack arms, which provides the driv- 
ing force to rotate the roller. The suspension system also provided about $50 \%$ reduction in the vertical displacement of the seat as the bicycle goes over a bump, which as well as providing a more comfortable ride also indicates that less energy is transferred from the roller to the bicycle during an impact.

The objective to establish a test configuration that would produce clear cut results that are not counter intuitive and where the physiological findings are reinforced by the mechanical measurements has been achieved. A basis has therefore been established for comparison with future experiments. These should be conducted under less constrained and less severe conditions with the aim of understanding better the effect of riding style and track conditions on performance and to provide data that can help optimise bicycle configurations and settings to achieve peak performance on designated types of terrain.

\section{Acknowledgements}

Our thanks go to Jon Whyte of Whyte Bicycles for the loan of the bicycles used in the tests. Thank you also to the Department of Mechanical Engineering and the Institute of Biomedical \& Life Sciences at the University of Glasgow for providing resources and technician support.

\section{References}

[1] Titlestad, J.: Mountain Bicycle Rear Suspension Dynamics and Their Effect on Cyclists. Thesis, The University of Glasgow, 2001.

[2] Seifert, J., Luetkemeier, M., Spencer, M., Miller, D., Burke, E.: The Effect of Mountain Bike Suspension Systems on the Energy Expenditure, Physical Exertion, and Time Trial Performance during Mountain Bicycling. International Journal of Sports Medicine, Vol. 18, 1997, p. 197-200.

[3] Wang, E. L., Hull, M. L.: A Model for Determining Rider Induced Energy Losses in Bicycle Suspension Systems. Vehicle System Dynamics, Vol. 25, 1996, p. 223-246.
[4] Berry, M. J., Woodard, C. M., Dunn, C. J., Edwards, D. G., Pittman, C. L.: The Effects of a Mountain Biking Suspension System on Metabolic Energy Expenditure. Cycling Science, 1993, p. 8-14.

[5] MacRae, H. S-H., Hise, K. J., Allen, P. J.: Effects of Front and Dual Suspension Mountain Bike Systems on Uphill Cycling Performance. Medicine and Science in Sports and Exercise, Vol. 32, 2000, p. 1276-1280.

[6] McArdle, W., Katch, F., Katch, V.: Exercise Physiology, Energy, Nutrition, and Human Performance. $4^{\text {th }}$ Edition, London: Williams \& Wilkins, 1996.

Figs. 1-4 are reproduced from reference [1]; copyright J.Titlestad

(B) This article includes words that are believed to be, or asserted to be, proprietary terms or trademarks. The authors recognise their status as such and no other judgement is implied concerning their legal status.

John Titlestad

Dr. Tony Fairlie-Clarke

phone: +441 413304327

e-mail: tonyfc@mech.gla.ac.uk

Mark Davie

Dr. Arthur Whittaker

Department of Mechanical Engineering

The University of Glasgow

Glasgow G12 8QQ, United Kingdom

Dr. Stan Grant

phone: +441413306490

e-mail: S.Granty@bio.gla.ac.uk

Institute of Biomedical \& Life Sciences

The University of Glasgow

Glasgow G12 8QQ, United Kingdom 